\title{
Faster Average Case Low Memory Semi-external Construction of the Burrows-Wheeler Transform
}

\author{
German Tischler
}

Received: 25 April 2014 / Accepted: 23 June 2014 / Published online: 24 March 2017

(C) The Author(s) 2017. This article is an open access publication

\begin{abstract}
The Burrows-Wheeler transform has applications in data compression as well as full text indexing. Despite its important applications and various existing algorithmic approaches the construction of the transform for large data sets is still challenging. In this paper we present a new semi external memory algorithm for constructing the Burrows-Wheeler transform. It is capable of constructing the transform for an input text of length $n$ over a finite alphabet in time $O\left(n \log ^{2} \log n\right)$ on average, if sufficient internal memory is available to hold a fixed fraction of the input text. In the worst case the run-time is $O(n \log n \log \log n)$. The amount of space used by the algorithm in external memory is $O(n)$ bits. Based on the serial version we also present a shared memory parallel algorithm running in time $O\left(\frac{n}{p} \max \left\{\log ^{2} \log n+\log p\right\}\right)$ on average when $p$ processors are available.
\end{abstract}

Keywords Burrows-Wheeler transform $\cdot$ Semi-external algorithm $\cdot$ Parallel index construction

Mathematics Subject Classification 68W32 (Algorithms on Strings) · 68W10 (Parallel Algorithms)

\section{Introduction}

The Burrows-Wheeler transform (BWT) was introduced to facilitate the lossless compression of data (cf. [3]). It has an intrinsic connection to some data structures used for full text indexing like the suffix array (cf. [19]) and is at the heart of some compressed full text self indexes like the FM index (see [9]). The FM index requires no more space than the k'th order entropy compressed input text plus some asymptotically negligible supporting data

${ }^{1}$ By $\log$ we mean $\log _{2}$ in this paper.

Supported by the Wellcome Trust.

Full version of an extended abstract which appeared in the Proceedings of the 2nd International Conference on Algorithms for Big Data.

G. Tischler $(\bowtie)$

The Wellcome Trust Sanger Institute, Wellcome Trust Genome Campus, Hinxton, Cambridge CB10 1SA, UK

e-mail: german.tischler@sanger.ac.uk

G. Tischler

Max Planck Institut für molekulare Zellbiologie und Genetik, Pfotenhauerstraße 108, 01307 Dresden, Germany

e-mail: tischler@mpi-cbg.de 
structures. Many construction algorithms for the BWT are based on its relation to the suffix array, which can be computed from the input text in time linear in the length of that text (see e.g. [13,22]). While these algorithms run in linear time and are thus theoretically optimal they require $O(n \log n)^{1}$ bits of space for the uncompressed suffix array given an input text of length $n$ while the text itself can be stored in a space of $n\lceil\log \sigma\rceil$ bits for an alphabet of size $\sigma$ where we often have $\sigma \ll n$ and in most applications $\sigma$ is constant. Algorithms for computing the suffix array in external memory have been proposed (see e.g. [2,6]) but these algorithms require large amounts of space and input/output in external memory. An asymptotically optimal internal memory solution concerning time and space has been proposed [11]. However the space usage of this algorithm is $O(n)$ bits for constant alphabets, where an inspection of the algorithm suggests that the actual practical memory usage of the algorithm is several times the size of the text in bits. The practical space usage of the algorithm subsequently presented in [23] is lower (i.e. the involved constants are smaller) while theoretically not linear. It however still requires multiple times as much space as the input text. A sample implementation given by the authors only works for input sizes of up to $2^{32}$ (see [1]) and only handles a single level of the recursive algorithm. Given the implementation complexity of the algorithm it remains unclear if it would scale well. Crochemore et al. present an algorithm computing the BWT in quadratic time with constant additional space (see [4]). Beller et al. [1] propose a semi external algorithm for the construction of the BWT based on induced sorting. An algorithm is called semi external if it uses non negligible amounts of internal as well as external memory. According to the authors the algorithm scales to arbitrary input sizes and uses about one byte (i.e. 8 bits) per input symbol in internal memory. An algorithm constructing the BWT of a text by block-wise merging using a finite amount of internal memory is presented in [8]. The algorithm partitions the text into a set of fixed size blocks. The run-time is $O\left(n^{2} / b\right)$ for a block size of $b$ and a text length of $n$. It requires an amount of internal memory which is roughly sufficient to compute the suffix array of a single of these block. In particular the amount of internal memory used can be smaller than the space required for the text. In this paper we modify this algorithm to run in time $O(n \log n \log \log n)$ in the worst case and $O\left(n \log ^{2} \log n\right)$ on average for the case where we are able to keep a fixed fraction of the text in memory. Assuming the practically common case of a finite alphabet the algorithm in [8] uses blocks of size $O(n / \log n)$ when provided with $O(n)$ bits of space in internal memory so its total run-time for this setting is $O(n \log n)$. In consequence our algorithm is faster on average and slower by $O(\log \log n)$ for a very unlikely worst case. Compared to the algorithm presented in [1] our algorithm can work with less internal memory. For DNA for instance the complete text can be held in memory using about 2 bits per symbol which is significantly less than a full byte $(8 \mathrm{bits})$ per character. We also propose a parallel version of our algorithm for the shared memory model. This algorithm runs in time $O\left(\frac{n}{p} \max \left\{\log ^{2} n, \log p\right\}\right)$ on average and with high probability when using $p$ processors.

The rest of this paper is structured as follows. In Sect. 2 we introduce definitions which we will be using throughout the paper. Section 3 gives a short overview over the algorithm of Ferragina et al. (cf. [8]) on which our new algorithm is based. In Sect. 4 we present an algorithm for computing the minimal periods of all prefixes of a string in succinct space. We show how to compute the suffix order for blocks of $b$ suffixes drawn from a circular version of a string of length $n \geq b$ in optimal time $O(b)$ and space $O(b \log b)$ bits after collecting some repetition information about the string in a preprocessing phase taking time $O(n)$ and $O(b \log b)$ bits of space in Sect. 5. In Sect. 6 we show how to merge the BWTs of two adjacent text blocks of length $b_{l}$ and $b_{r}$ respectively given by their BWT in external memory in time $O\left(\left(b_{l}+b_{r}\right) \log \log \left(b_{l}+b_{r}\right)\right)$ and sufficient space in internal memory to hold a rank dictionary for the left block. We describe in Sect. 7 how to combine the block sorting from Sect. 5 and block merging from Sect. 6 to obtain a BWT construction algorithm based on a balanced instead of a skewed merge tree. In Sect. 8 we describe a parallel version of our algorithm. Finally in Sect. 9 we summarise our results and discuss some remaining open problems.

\section{Definitions}

For a string $s=s_{0} s_{1} s_{2} \ldots s_{m-1}$ of length $|s|=m \in \mathbb{N}$ we define $s[i]=s_{i}$ for $0 \leq i<m$ and for $s=s_{0} s_{1} \ldots$ we define $s[i]=s_{i}$ for $0 \leq i$. For a finite word $u$ and a finite or infinite word $v$ we write their concatenation as $u v$. For any finite words $u, x$ and finite or infinite words $w, v$ such that $w=u x v$ we call $u$ a prefix, $v$ a suffix and $x$ a factor of $w$. A 
prefix, suffix or factor of some string $w$ is called proper if it is not identical with $w$. The empty word consisting of no symbols is denoted by $\epsilon$. For a string $s$ and indices $0 \leq i \leq j<|s|$ we denote the factor $s[i] s[i+1] \ldots s[j]$ by $s[i, j]$. For any $i, j$ such that $i>j$ the term $s[i, j]$ denotes the empty word. A finite word $w$ has period $p$ iff $w[i]=w[i+p]$ for $i=0, \ldots,|w|-p-1$ and an infinite word $w$ has period $p$ iff $w[i]=w[i+p]$ for $i=0,1, \ldots$ For a finite word $u$ and $k \in \mathbb{N}$ the $k^{\prime}$ th power $u^{k}$ of $u$ is defined by $u^{0}=\epsilon$ and $u^{i+1}=u^{i} u$ for $i \in \mathbb{N}$. A word $w$ is primitive if it is not a power of a word $u$ such that $|u|<|w|$. A word $u$ is a root of $w$ if $w=u^{k}$ for some $k \in \mathbb{N}$. A word $w$ is a square if there is a word $u$ such that $w=u^{2}$. Throughout this paper let $\Sigma=\{0,1, \ldots, \sigma-1\}$ denote a finite alphabet for some $\sigma \in$ $\mathbb{N}, \sigma>0$ and let $t=t_{0} t_{1} \ldots t_{n-1} \in \Sigma^{n}$ denote a finite string of length $n>0$. We define the semi infinite string $\tilde{t}$ by $\tilde{t}[i]=t[i-\lfloor i / n\rfloor n]$ for $i \geq 0$. We define the suffix $\tilde{t}_{i}$ of $\tilde{t}$ as $\tilde{t}_{i}=\tilde{t}[i] \tilde{t}[i+1] \ldots$ and $\tilde{t}_{i}<\tilde{t}_{j}$ for $i, j \in \mathbb{N}, i \neq j$ iff either $\tilde{t}_{i}=\tilde{t}_{j}$ and $i<j$ or for the smallest $\ell \geq 0$ such that $\tilde{t}[i+\ell] \neq \tilde{t}[j+\ell]$ we have $\tilde{t}[i+\ell]<\tilde{t}[j+\ell]$. The suffix array $A$ of $t$ is defined as the permutation of the numbers $0,1, \ldots, n-1$ such that $\tilde{t}_{A[i]}<\tilde{t}_{A[i+1]}$ for $i=0,1, \ldots, n-2$ and the Burrows-Wheeler transform (BWT) $B=b_{0} b_{1} \ldots b_{n-1}$ of $t$ is given by $b_{i}=\tilde{t}[A[i]+n-1]$ for $i=0,1, \ldots, n-1$.

\section{BWT Construction by Block-Wise Merging}

We give a short high level description of the algorithm by Ferragina et al. [8] as we will be modifying it. Unlike our algorithm it assumes the input string to have a unique minimal terminator symbol. Given a block size $b$ the input string $t$ is partitioned into $c=\lceil n / b\rceil$ blocks $T_{0}, T_{1}, \ldots, T_{c-1}$ of roughly equal size. The algorithm starts by suffix sorting the last block, computing its BWT $B_{c-1}$ and the bit array $g t_{c-1}$ which denotes for each suffix in $T_{c-1}$ but the first whether it is smaller or larger than the first. The BWT of $T_{i} \ldots T_{c-1}$ for $i<c-1$ is computed by first computing the suffix array for the suffixes starting in $T_{i}$ by using the text of $T_{i}$ and $T_{i+1}$ in memory and handling the comparison of suffixes starting in $T_{i}$ but equal until both have entered $T_{i+1}$ by using the bit vector $g t_{i+1}$ which explicitly stores the result of this comparison. The BWTs of $T_{i}$ and $T_{i+1} T_{i+2} \ldots T_{c-1}$ are merged by computing the ranks of the suffixes starting in $T_{i+1} T_{i+2} \ldots T_{c-1}$ in the sorted set of suffixes of $T_{i}$ and computing a gap array $G_{i}$ which denotes how many suffixes from $T_{i+1} T_{i+2} \ldots T_{c-1}$ are to be placed before the suffixes in $T_{i}$, between two adjacent suffixes in $T_{i}$ and after all suffixes in $T_{i}$. This process follows a backward search of $T_{i+1} T_{i+2} \ldots T_{c-1}$ in $T_{i}$. Using the array $G_{i}$ it is simple to merge the two BWTs together. For computing the rank of a suffix from $T_{i+1} \ldots T_{c-1}$ it is necessary to know whether it is smaller or larger than the one at the start of $T_{i+1} T_{i+2} \ldots T_{c-1}$ as $B_{i}$ is not a conventional BWT. For further details about the algorithm the reader is referred to [8].

\section{Computing the Minimal Period of the Prefixes of a String}

A border of a string $w$ is a string $u$ such that $w=u v=x u$ and $|v|=|x| \neq 0$. The border array $\mathcal{B}$ of a string $w$ is the integer array of length $|w|$ such that $\mathcal{B}[i]$ stores the length of the longest border of $w[0, i]$ for $i=0,1, \ldots,|w|-1$. When a string $w$ has the border array $\mathcal{B}$, then the minimal period of $w[0, i]$ for $0 \leq i<|w|$ can be obtained as $i+1-\mathcal{B}[i]$ (see e.g. Proposition 1.5 in [5]). The uncompressed border array for a string of length $b$ can be stored using $b$ words, i.e. $b\lceil\log b\rceil$ bits. Due to the properties of the border array (the value at index $i+1$ can be at most 1 larger than the value at index $i$ and all values are non-negative) it is however easy to use a succinct version in $O(b)$ bits. For the rest of the section let $w$ denote a string and let $\mathcal{B}$ denote the border array of $w$. Then we can represent $\mathcal{B}$ as the bit vector $\tilde{\mathcal{B}}=10^{\mathcal{B}[0]-\mathcal{B}[1]+1} 10^{\mathcal{B}[1]-\mathcal{B}[2]+1} 1 \ldots 0^{\mathcal{B}[b-1]-\mathcal{B}[b-2]+1} 1$ and $\mathcal{B}[i]$ can be retrieved as $i-\operatorname{rank}_{0}\left(\tilde{\mathcal{B}}, \operatorname{select}_{1}(\tilde{\mathcal{B}}, i)\right)$ for $i=0,1, \ldots, b-1$ where $\operatorname{rank}_{0}(\tilde{\mathcal{B}}, i)$ denotes the number of 0 bits in $\tilde{\mathcal{B}}$ up to and including index $i$ and $\operatorname{select}_{1}(\tilde{\mathcal{B}}, i)$ returns the index of the $i+1$ 'th 1 bit in $\tilde{\mathcal{B}}$. Indexes for the rank and select operations can be stored in $o(b)$ extra bits (see e.g. [21]). The most common indexes for these operations are tailored for static bit vectors while the computation of the border array (see for instance Sect. 1.6 in [5]) incrementally extends the array while using the already existing prefix to deduce the next value. The rank and select indexes as presented in Sect.6.1 in [21] are however easily adapted for the case of only appending bits at the end of the bit vector. Both types of indexes partition the indexed bit vector into blocks. In the case of rank the blocks consist of $\beta_{0}=\left\lceil\log ^{2}|\tilde{\mathcal{B}}|\right\rceil$ bits of $\tilde{\mathcal{B}}$. An array $\mathcal{R}_{0}$ is used to explicitly 
store $\operatorname{rank}_{0}\left(\tilde{\mathcal{B}}, \beta_{0} i\right)$ for $i=0,1, \ldots,\left\lceil\frac{n}{\beta_{0}}\right\rceil-1$ where each element of $\mathcal{R}_{0}$ takes $\lceil\log |\tilde{\mathcal{B}}|\rceil$ bits. The blocks are again partitioned into smaller blocks of length $\beta_{1}=\left\lceil\frac{\log |\tilde{\mathcal{B}}|}{2}\right\rceil$ (for the sake of simplicity in the discussion we assume $\beta_{1}$ divides $\beta_{0}$. The method is easily extended to the case where $\beta_{0}$ is not exactly a multiple of $\beta_{1}$ ). Another integer array $\mathcal{R}_{1}$ is used to store $\operatorname{rank}_{0}\left(\tilde{\mathcal{B}}, \beta_{1} i\right)-\operatorname{rank}_{0}\left(\tilde{\mathcal{B}},\left\lfloor\frac{\beta_{1} i}{\beta_{0}}\right\rfloor\right)$ for $i=0,1, \ldots,\left\lceil\frac{n}{\beta_{1}}\right\rceil-1$. Each element of $\mathcal{R}_{1}$ takes $\left\lceil\log \beta_{0}\right\rceil$ bits. We can incrementally build the rank dictionary by adding one value to $\mathcal{R}_{0}$ and $\left\lceil\frac{\beta_{0}}{\beta_{1}}\right\rceil$ values to $\mathcal{R}_{1}$ each time $\beta_{0}$ bits have been appended to the underlying bit vector. This can trivially be done in time $O\left(\beta_{0}\right)$ for each block of size $\beta_{0}$. The answers for a block yet to be completed can be stored explicitly in an array of size $\beta_{0}$ which takes $\beta_{0}\left\lceil\log \beta_{0}\right\rceil=O\left(\log ^{2} \tilde{\mathcal{B}} \log \tilde{\mathcal{B}}\right)$ bits and thus is asymptotically negligible compared to the space used for the bit vector itself. Using this method the rank operation can be computed in time $O(1)$ at any time and the time for adding each single bit to the vector is amortised constant. The method for keeping constant time select operations is conceptually very similar. The data structure partitions the bit vector $\tilde{\mathcal{B}}$ such that each block contains $\zeta_{0}=\left\lceil\log ^{2}|\tilde{\mathcal{B}}|\right\rceil$ bits of value 1. Again we can keep enough space to store the answers for a single incomplete block explicitly and extend the index for select each time the total number of 1 bits in the intermediate bit vector reaches an integer multiple of $\zeta_{0}$.

Lemma 1 Let $x$ denote a string over a finite alphabet $\Gamma=\{0,1, \ldots, \gamma-1\}$. The border array of $x$ can be computed in time $O(|x|)$ and using space $O(|x| \log \gamma)$ bits.

Lemma 2 Let $x$ denote a string over a finite alphabet $\Gamma=\{0,1, \ldots, \gamma-1\}$. The sequence of minimal periods of the prefixes of $x$ can be enumerated in time $O(|x|)$ and space $O(|x| \log \gamma)$ bits.

Using the periodicity lemma (see Lemma 1 in [17]) we in addition obtain the following lemma.

Lemma 3 Let $x$ denote a string over a finite alphabet $\Gamma=\{0,1, \ldots, \gamma-1\}$. If $x$ has any period $q \leq\left\lfloor\frac{|x|}{2}\right\rfloor$ then there exists a minimal period $p$ of $x$ such that $p \leq\left\lfloor\frac{|x|}{2}\right\rfloor$ and $p$ divides all other periods of $x$ whose value does not exceed $\left\lfloor\frac{\lfloor x \mid}{2}\right\rfloor$. It is decidable in time $O(|x|)$ and space $O(x \log \gamma)$ bits whether $x$ has any period $p \leq\left\lfloor\frac{|x|}{2}\right\rfloor$ and if it has any such periods then their minimum can be computed in the same time and space bounds.

Proof Let $x$ denote a string over a finite alphabet $\Gamma=\{0,1, \ldots, \gamma-1\}$ and let $P$ denote the set of all periods of $x$ whose value does not exceed $\left\lfloor\frac{|x|}{2}\right\rfloor$. If $P$ is empty, then the lemma holds as $x$ has no relevant periods. Otherwise let $p$ be the minimal element of $P$. Then for any element $q$ of $P$ we have $p+q \leq|x|$ as $p \leq q \leq\left\lfloor\frac{|x|}{2}\right\rfloor$. According to the periodicity lemma this implies that the greatest common divisor of $p$ and $q$ is also a period of $x$. As $P$ contains all the periods of $x$ not exceeding $\left\lfloor\frac{|x|}{2}\right\rfloor, p$ is the minimal element of $P$ and the greatest common divisor of $p$ and $q$ divides $p$ it follows that this greatest common divisor is $p$. As $p$ is the minimal period of $x$ it can be computed in time $O(|x|)$ and space $O(|x| \log \gamma)$ bits according to Lemma 2.

\section{Sorting Single Blocks}

The algorithm by Ferragina et al. processes each single block relying on knowledge about the priorly fully processed following block, in case of the last block the terminator. For our algorithm we need to be able to sort a single block without knowing the complete sorted order of the next block. For this purpose we need to be able to handle repetitions, one of the major challenges along the way, efficiently. For the following argumentation we will need the occurring block sizes to be as similar as possible, so we will deduce a final block size from a target block size chosen to satisfy memory constraints. Let $b^{\prime}$ denote a preliminary target block size such that $0<b^{\prime} \leq n$. From $b^{\prime}$ we can deduce a final block size $b$ as

$b=\left\lceil\frac{n}{\left\lceil\frac{n}{b^{\prime}}\right\rceil}\right.$.

In Eq. 1 the denominator denotes the number $v$ of blocks required for the preliminary block size $b^{\prime}$. The final block size is obtained as the smallest number $b$ of symbols required to keep this number of blocks $v$, in particular we have 
$b \leq b^{\prime}$ As $b$ is the smallest such number we have $\mu^{\prime}=v b-n$ such that $0 \leq \mu^{\prime}<v$ and equally $\mu=v-\mu^{\prime}$ such that $1 \leq \mu \leq v$. In consequence we can decompose the set of indices $[0, n)$ on $t$ into $\mu$ sub intervals of length $b$ and $v-\mu$ sub intervals of length $b-1$ and define the index blocks $\mathcal{B}_{i}$ by

$\mathcal{B}_{i}=\left\{\begin{array}{lr}{[i b,(i+1) b)} & \text { for } 0 \leq i<\mu \\ {[\mu b+(i-\mu)(b-1), \mu b+(i+1-\mu)(b-1)} & \text { for } \mu \leq i<v\end{array}\right.$

Informally we have $\mu$ blocks of size $b$ and $v-\mu$ blocks of size $b-1$ such that the blocks of size $b$ precede the ones of size $b-1$. Blocks of size $b-1$ only exist if $b$ does not divide $n$.

When comparing two suffixes of $\tilde{t}$ starting at indices $i$ and $j$ such that $i<j$ we may encounter two cases. In the first case we have $\tilde{t}[i, j-1] \neq \tilde{t}[j, j+(j-i-1)]$. Then the comparison requires handling at most $j-i$ symbol pairs, i.e. at most $2 b-2$ symbols if the two suffixes start in the same block of length $b$ as defined above. In the second case we have $\tilde{t}[i, j-1]=\tilde{t}[j, j+(j-i-1)]$, i.e. a square with period $j-i$ at index $i$ in $\tilde{t}$. Thus situations which require us to have access to $\omega(b)$ symbols for sorting the suffixes of a block of indices on $\tilde{t}$ are induced by repetitions of periods strictly smaller than $b$. In consequence only repetitions with a period smaller than $b$ are relevant for our block sorting.

Definition 1 Let $\mathcal{B}$ denote a block of $b$ indices on $\tilde{t}$ starting at index $i$, i.e. the set of suffixes $\tilde{t}_{i+j}$ for $j=$ $0,1, \ldots, b-1$.

- $\mathcal{B}$ propagates a repetition of period $p, 1 \leq p \leq b$ iff $\tilde{t}_{i}[0, b+2 p-1]$ has period $p$.

- $\mathcal{B}$ generates a repetition of period $p, 1 \leq p \leq b$ iff $\tilde{t}[b-p, b-1]=\tilde{t}[b, b+p-1]$ and the block of $b$ suffixes starting at $i+b$ propagates a repetition of period $p$.

If a block propagates repetitions of any periods, then there is a unique minimal period dividing all other propagated periods according to Lemma 3. This unique minimal period can then be computed in time $O(b)$ and space $O(b \log \sigma)$ bits using minor modifications of standard string algorithms (see Sect.4). As there is a unique minimal period propagated by a block if any and as for repetition generation we are only interested in periods which are propagated by the next block we can compute the relevant generation properties of a block in the same time and space bounds. The suffix order on $\tilde{t}$ defined as above defines the order of two suffixes at indices $i$ and $j$ such that $i \neq j$ by index comparison if $\tilde{t}_{i}=\tilde{t}_{j}$. This case can only appear if the text $t$ itself has a period $p$ which is smaller than $n$ and divides $n$, i.e. when $t=\alpha^{i}$ for some string $\alpha$ and some integer $i>1$. For sorting the suffixes in our blocks we can avoid this case. If $t$ is an integer power of a string $\alpha$ such that $|\alpha|<b$ for some exponent $k>1$, then we will observe that each of our blocks propagates period $p$, thus this case is easily detected given precomputed repetition propagation information. In this case the Burrows-Wheeler transform of $t$ can be obtained from the Burrows-Wheeler transform for $\alpha$ by repeating each symbol $k$ times, e.g. if $a b c$ is the Burrows-Wheeler transform for $\alpha$ and we have $k=2$ then the transform for $t$ is $a a b b c c$. Similar arguments can be employed to obtain the suffix array and inverse suffix array of $t$ from the reduced variants for $\alpha$. Thus in the following we will without loss of generality assume that $t$ is not an integer power of a string of period smaller than $b$. In consequence all suffix comparisons required for sorting the suffixes starting in a block are decided by character inequality and not by position. Using information about short period repetitions in the input string, we are able to handle the sorting of a single block of suffixes extending beyond the end of the block efficiently by reducing long repetitions as we show in the following lemma.

Lemma 4 A block of b circular suffixes of $\tilde{t}$ can be sorted in lexicographical order using time $O(b)$ and space $O(b \log b)$ bits using precomputed repetition propagation data.

Proof The pairwise order between all suffixes of the block is decided by character inequalities. However these character inequalities may happen after $\Omega(n)$ symbols in each case. Note however that if we compare two strings $u$ and $v$ lexicographically such that $u=\alpha \beta^{k} \gamma$ and $v=\alpha \beta^{k} \delta$ we obtain the same result as when comparing $u^{\prime}=\alpha \gamma$ and $v^{\prime}=\alpha \delta$, i.e. as when we remove the repetition of $\beta$ from both. Now assume we are sorting the circular suffixes of $t$ for a block starting at index $i$ with block length $b$, i.e. we are sorting $b$ strings of infinite length where for each 
pair of strings the comparison ends after less than $n$ steps. When comparing a pair of such strings starting at offsets $j$ and $k$ in the block such that without loss of generality $j<k$ we may encounter the following cases.

1. There exists $\ell<(b-j)+(k-j)=b+k-2 j$ such that $\tilde{t}[i+j+\ell] \neq \tilde{t}[i+k+\ell]$. Then as $b+k-2 j<2 b$ the order between the strings would be correctly reflected in a suffix sorting of $\tilde{t}[i, i+2 b-1]$, where $2 b$ is $O(b)$.

2. Otherwise we have $\tilde{t}[i+j, i+k-1]=\tilde{t}[i+k, i+k+(k-j)-1]$, i.e. a square of period $k-j$ at position $i+j$ in $\tilde{t}$ and this repetition extends to at least index $i+b+(k+j)-1$ in $\tilde{t}$. There is thus a complete instance of a rotation of the root of the square in $\tilde{t}$ after the end of the considered block (the root of the repetition may overlap the block boundary or come to lie outside of the block). In consequence the block may generate a repetition of period $k-j$ depending on whether the following block propagates a repetition of this period. If $\tilde{t}[i+j, i+k-1]$ is an integer power of a shorter primitive string $u$, then let $p=|u|$, otherwise let $p=k-j$. Now we may encounter two sub cases. If the following block does not propagate period $p$, then the order between our two strings starting at index $i+j$ and $i+k$ in $\tilde{t}$ would be correctly reflected in a suffix sorting of $\tilde{t}[i, i+2 b+2 p-1]$ due to the definition of the propagation of a repetition. Note that $2 b+2 p-1$ is $O(b)$ as $p \leq k-j<b$. Now assume the following block does propagate period $p$. Let $\beta=\tilde{t}[i+b, i+b+p-1]$ and let $\alpha=\tilde{t}[i, i+(b-p)-1]$. Then the block can be written as $\alpha \beta$. Further we know that $\tilde{t}[i] \tilde{t}[i+1] \ldots=\alpha \beta^{m} \gamma$ for some integer $m>1+\lceil b / p\rceil$ and $\gamma[0, p-1] \neq \beta$. Using precomputed information about the single blocks' repetition propagation properties and adequate data structures it is simple to deduce the numbers $m$ and $\gamma$ in time $O(b)$. For each block propagating a repetition we store the index of the closest following block in $\tilde{t}$ which no longer propagates this repetition in an array. This array can be computed in time $O(n)$ with a single scan over the list of repetitions propagated by the single blocks. Even for a moderate block size in $O\left(\log ^{2} n\right)$ the space required for this array is asymptotically negligible compared to the text and it can be stored in external memory. Now for computing $m$ and $\gamma$ we can go to the last following block still propagating the repetition and perform a naive scan of the text until the repetition ends. This scan will terminate in time $O(b)$. For determining the order of the suffixes in the current block it is sufficient to sort $\alpha \beta^{1+\lceil b / p\rceil} \gamma$ which has length $O(b)$.

Sorting the suffixes of $\tilde{t}$ starting in a given block of length $b$ using the precomputed repetition generation and propagation block properties can thus be performed in time $O(b)$ using space $O(b \log b)$ bits in internal memory. Given the explicit suffix sorting for a block, it is trivial to determine, whether any other suffix in the block is lexicographically smaller or larger than the first suffix of the block and store the resulting bit vector in external memory.

For forward searching using the suffix array it is useful to in addition have the longest common prefix (LCP)

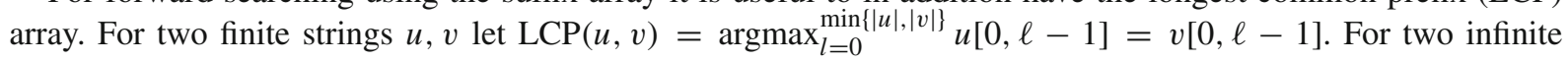
strings $u, v$ let $\operatorname{LCP}(u, v)=\infty$ if $u=v$ and $\operatorname{LCP}(u, v)=i$ where $i$ is the smallest non negative integer such that $u[i] \neq v[i]$ otherwise. For a block of indices $[i, i+b-1]$ on $\tilde{t}$ such that $i \in \mathbb{N}$ let $\mathcal{A}$ denote the permutation of $i, i+1, \ldots, i+b-1$ such that $\tilde{t}_{\mathcal{A}[j]}<\tilde{t}_{\mathcal{A}[j+1]}$ for $j=0,1, \ldots, b-2$. Then the LCP array of the block is defined by $\operatorname{LCP}[0]=0$ and $\operatorname{LCP}[i]=\operatorname{LCP}\left(\tilde{t}_{\mathcal{A}[i-1]}, \tilde{t}_{\mathcal{A}[i]}\right)$ for $i=1,2, \ldots, b-1$. Using a repetition reduction method similar to the suffix sorting case we obtain the following result.

Lemma 5 The LCP array for a block of b circular suffixes on $\tilde{t}$ can be computed in time $O(b)$ and space $O(b \log b)$ bits using precomputed repetition propagation data.

Proof As the input string is without loss of generality not an integer power of a string of period $p<b$ all suffix comparisons on $\tilde{t}$ within a block are decided by character inequalities. In consequence the values in the LCP array of a block are finite but the array can contain values in $\Omega(n)$ as the considered suffixes extend beyond the end of the block. Given a finite string $s$ and the suffix array of $s$ the LCP array for $s$ can be computed in time $O(|s|)$ and stored in an array of size $O(s \log s)$ bits (see [16]). Thus if the block does not generate a repetition, then we can compute the LCP array for the block in time $O(b)$ and represent it as an array using $O(b \log b)$ bits. This involves extending the text block by $O(b)$ elements (see above), computing the LCP array using the suffix array and the text and filtering the LCP array so that only suffixes inside the original block remain. If we find suffixes outside the original block between two original suffixes than we need to combine the minimum of 
the corresponding entries with the second original block value, e.g. if the block starts at index $i$ and we have $\mathcal{A}[j]<i+b, \mathcal{A}[j+1] \geq i+b, \mathcal{A}[j+2] \geq i+b, \ldots, \mathcal{A}[j+k]<i+b$ then the LCP value between the suffixes at the block indices $\mathcal{A}[j]$ and $\mathcal{A}[j+k]$ is $\min \{\operatorname{LCP}[j+1], \operatorname{LCP}[j+2], \ldots, \operatorname{LCP}[j+k]\}$ and we drop the LCP values relating to suffixes outside the block. Now assume the block generates a repetition of period $p$. Then as described in case 2 of the proof of Lemma 4 it is sufficient to suffix sort the string $\alpha \beta^{1+\lceil b / p\rceil} \gamma$ as defined above to obtain the order of the suffixes in the current block. This block has length $O(b)$, so its LCP array can be computed in time $O(b)$ and stored in space $O(b \log b)$ bits. We first remove the values corresponding to suffixes outside the block as we did for the non propagating case. Then the LCP value at index $j>1$ is correct if $\operatorname{LCP}[j]<\min \{i+b-\mathcal{A}[j-1], i+b-\mathcal{A}[j]\}$, i.e. if the LCP between the two corresponding suffixes is too short to reach the next block for at least one of the two. Otherwise both suffixes are equal at least until the comparison of the two has extended to the next block. Then the stored LCP value will be too small and thus incorrect. We can however easily correct these values using the precomputed repetition propagation information. We can compute in time $O(b)$ at which offset from the start of the next block the propagated repetition first breaks. Let $o$ denote this offset. Then we obtain the correct LCP value for index $j>0$ as $\operatorname{LCP}[j]=(i+b-\max \{\mathcal{A}[j-1], \mathcal{A}[j]\})+o$. If we store this value explicitly, then the LCP array will take space $O(b \log n)$ bits. As we can however detect and correct the incorrect LCP values in constant time given the suffix array and number $o$ we can leave the LCP array as is taking space $O(b \log b)$ and still have constant time access to the correct values by computing them as required.

Using the suffix and LCP array the time for a forward search of a pattern of length $m$ in a block of size $b$ reduces from $O(m \log b)$ to $O(m+\log b)$ (see [19]).

\section{Merging Pairs of Adjacent Blocks}

In our modified algorithm we replace the completely skewed binary merge tree used in [8] by a balanced binary merge tree. Consequently we will need to be able to merge blocks with a block size in $\Omega(n)$. For merging two adjacent blocks we need the following components:

1. The BWT of the left and right block. These can be compressed and in external memory as they will be scanned sequentially.

2. An internal memory index of the left block suitable for backward search in $O(1)$ time per step. An FM type index using space $b_{l} H_{k}+o(n \log \sigma)$ bits can be used where $b_{l}$ is the length of the left block and $H_{k}$ denotes the k'th order entropy of the left block (see [21]).

3. The $g t$ bit vectors for the left and right block. Scanned sequentially and thus can be read streaming from external memory.

4. The number of circular suffixes in the left block smaller than the rightmost suffix of the right block. Used as the start point for the backward search.

5. The gap array $G$.

The first three are equivalent to those used in [8]. The rank of the rightmost suffix in the right block relative to the suffixes of the left block can be obtained by employing forward search on one or more text blocks. If the left block is a single block which was produced by explicit suffix sorting using the method of Sect. 5, then the rank can be obtained using classical forward search in the suffix array while using the adjoined LCP array. This takes time $O(n+\log b)$ in the worst case (on average this can be expected to be $O(\log n+\log b)$, see [25]). If the left block was already obtained by merging $c$ blocks together, then the desired rank can be obtained as the sum of the ranks of the suffix relative to all single blocks composing the left block in time $O(c(n+\log b))$. Assuming the blocks are merged together in a balanced binary merge tree the total time used for forward searches is $O\left(\frac{n}{b} \log \frac{n}{b} n\right)$ in the worst case and $O\left(\frac{n}{b} \log \frac{n}{b} \log n\right)$ on average. If we choose $b \in O\left(\frac{n}{\log n}\right)$ then this becomes $O(n \log n \log \log n)$. The memory required for the index of the left block in internal memory will be $b_{l}\lceil\log \sigma\rceil+o\left(b_{l} \log \sigma\right)$ bits for a left block size of $b_{l}$ assuming that the entropy compression is ineffective. This leaves us with the space required for 
the gap array. In the original algorithm this is a conventional array in internal memory taking space $O(b \log n)$ bits for a left block size of $b$. As we want to be able to merge blocks with size in $\Omega(n)$ this space requirement is too high. Using Elias $\gamma$ code (cf. [7]) we can store the gap array for merging a left and right block of length $b_{l}$ and $b_{r}$ respectively in $O\left(b_{l}+b_{r}\right)$ bits of space as we show in the following lemma.

Lemma 6 Let $G$ denote an array of length $\ell$ such that $G[i] \in \mathbb{N}$ for $0 \leq i<\ell$ and $\sum_{i=0}^{l-1} G[i]=s$ for some $s \in \mathbb{N}$. Then the $\gamma$ code for $G$ takes $O(\ell+s)$ bits.

Proof Elias $\gamma$ code stores the number $z>0$ as the bit sequence $0^{\lfloor\log z\rfloor} \operatorname{bin}(z)$ of length $1+2\lfloor\log z\rfloor$ where $\operatorname{bin}(z)$ denotes the binary representation of $z$ (most significant to least significant bit left to right). The $\gamma$ code for $G$ thus requires

$\ell+2 \sum_{i=0}^{\ell-1}\lfloor\log (G[i]+1)\rfloor$

bits. As $\gamma$ code is unable to represent the number zero but zero is a valid entry in $G$ we add 1 to each element. We have $\sum_{i=0}^{b_{l}-1}\lfloor\log (G[i]+1)\rfloor \leq \sum_{i=0}^{b_{l}-1} \log (G[i]+1)=\log \prod_{i=0}^{b_{l}-1}(G[i]+1)$.

and the last product is maximal for $G[0]=G[1]=\cdots=G[\ell-1] \approx \frac{s}{\ell}$ (conceptually due to the fact that $x(1-x$ ) has its maximum at $x=(1-x)=\frac{1}{2}$, i.e. a product of two positive real numbers of constant sum is maximal if both are chosen as half the sum). Thus the maximum space used for the gap array in $\gamma$ code is bounded by

$\ell+2 \sum_{i=0}^{\ell-1}\lfloor\log G[i]+1\rfloor \leq \ell\left(1+2 \log \left(\frac{s}{\ell}+1\right)\right)$

For $s \leq \ell$ the argument of the logarithm is between 1 and 2 thus the space usage is bounded by $3 \ell$. For $s>\ell$ let $s=2^{r} l$ for some $r \in \mathbb{R}, r>0$. Then we have

$$
\begin{aligned}
\ell\left(1+2 \log \left(\frac{s}{l}+1\right)\right) & \leq \ell\left(1+2+2 \log \frac{s}{l}\right) \\
& \leq \frac{s}{2^{r}}(3+2 r)=s \frac{3+2 r}{2^{r}} \leq s\left(3+\frac{1}{\log _{e} 2}\right) \leq 5 s
\end{aligned}
$$

which is in $O(s)$. Thus in both cases the space is in $O(\ell+s)$ bits.

Elias $\gamma$ code however is not suitable for efficient updating as we would need it for computing the gap array. During the computation of a gap array each step of the backward search leads to an increment of exactly one position in the gap array. In particular the sum over the elements of the array after $s$ steps is $s$. The $\gamma$ code representation of the gap array does not allow efficient updating of single elements. We overcome this problem by producing partial sparse gap arrays, which we write to external memory. We accumulate an amount $c$ of indices for incrementing in internal memory before we write a sparse variant to external memory, which implies that the sum over the sparse array written will be $c$. Whenever we have produced two sparse gap arrays in external memory featuring the same element sum, then we merge the two together into a sparse array of twice that sum. At the end of the process we need to merge the remaining set of arrays into a single final gap array. Let $G$ denote a gap array of length $\ell$ such that $\sum_{i=0}^{\ell-1} G[i]=s$ for some $s>0$ with $k>0$ non-zero values. Let $i_{0}, i_{1}, \ldots, i_{k-1}$ denote the sequence of indices of non-zero values in $G$ in increasing order. We store two $\gamma$ coded bit streams for representing $G$. In the first we store the sequence $i_{0}+1, i_{1}-i_{0}, i_{2}-i_{1}, \ldots, i_{k-1}-i_{k-2}$ and in the second we store the subsequence of non-zero values in $G$. Note that the elements of both sequences are strictly positive integers, so we do not need to add 1 for the $\gamma$ code. The maximum space usage for the first sequence is bounded by $k\left(1+2 \log \frac{\ell}{k}\right)$ and for the second by $k\left(1+2 \log \frac{s}{k}\right)$ which sums up to $2 k\left(1+\log \frac{s \ell}{k^{2}}\right)$ ) (see the proof for Lemma 6 for details on obtaining these bounds). For fixed $s, \ell$ this function has a maximum for $k=\frac{\sqrt{2 s \ell}}{e}$ with value $\frac{4 \sqrt{2 s \ell}}{e \log _{e} 2}$ where $e$ denotes the base of the natural 
logarithm. As we by definition have $k \leq \min \{\ell, s\}$ this maximum can only be observed for $s \geq \frac{2 \ell}{e^{2}} \approx .2706 \ell$ (and equally $\ell \geq \frac{2 s}{e^{2}}$ ). For $s<\frac{2 \ell}{e^{2}}$ we obtain the maximum space usage for $k=s$ and this space maximum is then bounded by $2 s\left(1+\log \frac{\ell}{s}\right)$. While computing a complete gap array we may at an intermediate stage have no more than two arrays with a sum of $c 2^{i}$ for $i=0,1, \ldots,\left\lfloor\log \frac{s}{c}\right\rfloor$ when the arrays of the smallest sum we produce have sum $c$ for a constant $c \geq 1$. We will never have two arrays for more then two of the sizes while merging and only a single one for all other sizes, but for the sake of simplicity in obtaining a bound on the size of the sum of the arrays we will assume that two for each size are possible. For those arrays with a sum of at most $\frac{\ell}{4}$ we can bound the space used by $\begin{aligned} 2 \sum_{i=0}^{\left\lfloor\log \frac{l}{4 c}\right\rfloor} 2\left(2^{i} c\right)\left(1+\log \frac{\ell}{2^{i} c}\right) & \leq 4 c \sum_{i=0}^{\left\lfloor\log \frac{l}{4 c}\right\rfloor} 2^{i}\left(1+\log \frac{\ell}{2^{i} c}\right) \\ & \leq 4 c \sum_{i=0}^{\left\lfloor\log \frac{\ell}{4 c}\right\rfloor} 2^{i}\left(1+\log \frac{\ell}{2^{i}}\right) \\ & \leq 8 c \sum_{i=0}^{\left\lfloor\log \frac{\ell}{4 c}\right\rfloor} 2^{i}\left(\log \frac{\ell}{2^{i}}\right) \\ & \leq 8 c \sum_{i=0}^{\left.\log \frac{\ell}{4 c}\right\rfloor} 2^{i}(\log \ell-i) \\ & \leq 8 c \sum_{i=0}^{\lceil\log \ell\rceil} 2^{i}(\lceil\log \ell\rceil-i) \\ & \leq 2^{\lceil\log \ell\rceil+3} c \sum_{i=0}^{\lceil\log \ell\rceil} \frac{i}{2^{i}} \\ & \leq 2^{\lceil\log \ell\rceil+4} c \\ & \leq 32 c \ell\end{aligned}$

Thus the space for these arrays is $O(\ell)$ bits as $c$ is a constant. For partial arrays with a sum of more than $\frac{\ell}{4}$ we can resort to dense arrays. We may have a constant number of arrays with a sum between $\frac{\ell}{4}$ and $\ell$. These take space $O(\ell)$ (see the proof for Lemma 6). Finally we can have a logarithmic number of arrays with a sum larger then $\ell$. Analogously to the arrays with a sum of at most $\frac{\ell}{4}$ we can deduce that the space for these arrays is $O(s)$ bits. In total all partial gap arrays together take space $O(s+\ell)$ bits. Two sparse partial gap arrays can be merged in time linear in the number of their respective non-zero elements which is bounded by the sum over the elements. Two of the dense partial gap arrays we use can also be merged in time linear in the sum over their elements, as these arrays are only used if the sum over their elements reaches a quarter of there length. Merging two partial gap arrays is in both cases a streaming operation and requires virtually no internal memory. If the merging process starts with partial arrays for sum 1, the final array has length $\ell$ and the sum over its elements is $s$ then the total merging time can be bounded by $\sum_{i=0}^{\lceil\log s\rceil} \min \{s, \ell\}$. For merging two blocks of size $b_{l}$ and $b_{r}$ respectively this is in $O\left(\min \left\{b_{l}, b_{r}\right\} \log \max \left\{b_{l}, b_{r}\right\}\right)$ which becomes $O\left(b_{l} \log b_{l}\right)$ if $b_{l} \in \Theta\left(b_{r}\right)$ as it holds for balanced binary merge trees. If we accumulate $\frac{b_{r}}{\log ^{2} b_{r}}$ indices for incrementing in an array in internal memory we can reduce the time used for merging without asymptotically increasing the total memory footprint of the algorithm. The space used for the array in internal memory is $O\left(\frac{b_{r}}{\log b_{r}}\right)$ bits and thus asymptotically negligible compared with the (uncompressed) text. When the internal memory array is full, then we first need to sort it so we can write the partial gap array representation. This can be performed in time linear in the length of the array by using a two phase radix sort with a number of buckets in $O\left(\sqrt{b_{r}}\right)$, which require additional internal memory of $O\left(\sqrt{b_{r}}\right)$ words or $O\left(\sqrt{b_{r}} \log b_{r}\right)$ bits. For both run-time and space usage the radix sort does not asymptotically increase the resources required for the merging. Starting with partial gap arrays of sum $\frac{b_{r}}{\log ^{2} b_{r}}$ implies the total number of such arrays is $O\left(\log ^{2} b_{r}\right)$. Consequently the binary merge tree for obtaining the final gap array has a depth in $O\left(\log \log ^{2} b_{r}\right)=O\left(\log \log b_{r}\right)$ and it can be processed in time $O\left(b_{r} \log \log b_{r}\right)$.

The $g t$ array for the merged block can be composed by concatenating the $g t$ array for the left block and an array storing the respective information for the right block computed while performing the backward search for filling the gap array. For this purpose we need to know the rank of the leftmost suffix in the left block. This can either be computed using forward search on the suffix arrays of the basic blocks or extracted from a sampled inverse suffix array which can be computed along the way. The sampled inverse suffix arrays of two blocks can just like the BWTs 
of the two blocks be merged using the gap array. This is also an operation based on stream accesses, so it can be done in external memory in time $O\left(b_{l}+b_{r}\right)$ when merging two blocks of size $b_{l}$ and $b_{r}$.

\section{BWT Computation by Balanced Tree Block Merging}

Using the building blocks described above we can now describe the complete algorithm for computing the BWT of $t$ by merging basic blocks according to a balanced binary tree.

1. Choose a target block size $b^{\prime} \in O\left(\frac{n}{\log n}\right)$ and deduce a block size $b=\left\lceil\frac{n}{\left\lceil\frac{n}{b^{\prime}}\right\rceil}\right\rceil$ such that the number of blocks $c$ satisfies $c=\left\lceil\frac{n}{b}\right\rceil=\left\lceil\frac{n}{b^{\prime}}\right\rceil$ and $n$ can be split into blocks of size $b$ and $b-1$ only. Split $t$ such that the blocks of size $b$ appear before those of size $b^{\prime}$. This step takes constant space and time.

2. Compute which blocks in $t$ propagate repetitions of period at most $b$ and for each block which is followed by a block propagating a repetition whether it is generating this repetition. This takes time $O(n)$ in total and space $O(b \log \sigma)=O\left(\frac{n \log \sigma}{\log n}\right) \subseteq O(n)$ bits. The result data can be stored in external memory.

3. Compute a balanced merge tree for the blocks. Start with a root representing all blocks. If a node containing a single block is considered produce a leaf and stop. Otherwise for an inner node representing $k>1$ blocks produce a left subtree from the $\left\lceil\frac{k}{2}\right\rceil$ leftmost blocks and a right subtree from $\left\lfloor\frac{k}{2}\right\rfloor$ rightmost blocks in $t$. The tree has $O(\log n)$ nodes. Each node stores at most two (start and end) block indices taking $O(\log \log n)$ bits and two node pointers also taking space $O(\log \log n)$ bits. So the total tree takes space $O(\log n \log \log n)$ bits. It can be computed in time $O(\log n)$.

4. Sort the blocks and store the resulting BWT, $g t$ and sampled inverse suffix arrays in external memory. Using the suffix and LCP arrays of the basic blocks also compute the start ranks necessary for the backward searches when merging the blocks together. This takes time $O(n \log n \log \log n)$ in the worst case and $O(n)$ on average and space $O(b \log b)=O\left(\frac{n}{\log n} \log \frac{n}{\log n}\right)=O(n)$ bits of internal memory.

5. Process the merge tree. Mark all leafs as finished and all inner nodes as unfinished. While there are unfinished nodes choose any unfinished node with only finished children, merge the respective blocks and mark the node as finished. There are $O(\log n)$ leafs and the tree is balanced, so it has $O(\log \log n)$ levels. Each single level can be processed in time $O(n \log \log n)$. So the total run time for the tree merging phase is $O\left(n \log ^{2} \log n\right)$. The maximum internal memory space usage appears when performing the merge operation at the root of the tree. Here we need space $b_{l} H_{k}+o\left(b_{l} \log \sigma\right)$ bits where $b_{l}$ denotes the sum of the length of the blocks in the left subtree which is $O(n)$ and $H_{k}$ denotes the $k$ 'th order entropy of the text comprising those text blocks.

Summing over all steps the run-time of the algorithm is $O(n \log n \log \log n)$ in the worst case and $O\left(n \log ^{2} \log n\right)$ on average. In practice this means we can compute the BWT of a text as long as we are able to hold the text (more precisely the text for the left subtree of the merge tree) in internal memory. If we can hold a fixed fraction of the text in main memory, then we can still compute the BWT of the text in the same run-time by resorting to the original iterative merging scheme from [8]. We decompose the text into blocks of size $b^{\prime}$ such that $b^{\prime} \leq \frac{n \log \sigma}{c \log n}$ where $\frac{1}{c}$ is the fixed fraction of the text we can hold in internal memory and compute a partial BWT for each of these blocks where the suffixes sorted are considered as coming from the whole text, i.e. suffix comparisons are still over $\tilde{t}$ and not limited to a single of the blocks. Then we merge these blocks along a totally skewed merge tree such that the left block always has size about $b^{\prime}$. The size of the set of partial sparse gap arrays required at any time remains bounded by $O(n)$ bits. As the number of blocks is fixed, the total asymptotical run-time of the algorithm remains $O(n \log n \log \log n)$ in the worst case and $O\left(n \log ^{2} \log n\right)$ on average.

\section{Parallelisation}

Many of the steps of our algorithm are parallelisable. As building blocks we will need representations of the required data structures in external memory which allow accessing parts without performing a complete sequential scan and we will require parallel versions of 
- the construction of rank indexes,

- the computation of gap arrays,

- the merging of BWT blocks,

- the merging of sampled inverse suffix arrays and

- the sorting of single blocks.

As the merging of sampled (inverse) suffix arrays is basically a simplified version of the merging of BWT blocks (the difference is not all values are present), we will only describe the merging of BWT blocks and leave the sub sampled merging variant as an exercise for the reader. In this paper we consider shared memory parallelisation only, i.e. a setting where all processors involved have access to the same internal memory. Some of the algorithms may be modified for distributed memory settings.

\subsection{Data Structures in External Memory}

We are using the following data structures in external memory:

- The $g t$ bit vectors

- Burrows-Wheeler transforms of blocks

- Dense $\gamma$ coded gap arrays

- Sparse $\gamma$ coded gap arrays

Arbitrary positions in the $g t$ bit vectors can be accessed without further information, so no additional information is necessary to support scanning starting from a given position.

For the Burrows-Wheeler transform sequences we choose the following representation. For a given string $B=b_{0} \ldots b_{m-1}$ over $\Sigma$ and a given block size $d \in O\left(\log ^{2} n\right)$ we partition $B$ into blocks of size $d$ such that all but the last block have size $d$. Each block factor is stored using run-length, Huffman and $\gamma$ code. We first compute a run length representation of the block which gives us a sequence of pairs comprised of symbols and number of consecutive occurrences. As an example the factor a abbbcaaa would be transformed to $(a, 2),(b, 3),(c, 1),(a, 3)$. This sequence of pairs is stored using Huffman code for the symbols and Elias $\gamma$ code for the run lengths. As we assume a constant alphabet size, the dictionary for the Huffman code takes constant space per block. The space used by the Huffman coded sequence of symbols is bounded by $d\lceil\log \sigma\rceil$. According to our considerations about $\gamma$ code above the maximum amount of space required for storing any sequence of run lengths for a block of length $d$ is $d(1+2\lceil\log (1+1)\rceil)=3 d$. So in the worst case the representation of the block BWT in this code is in $O(d \log \sigma)$ bits. In consequence each block can be addressed using a fixed size pointer of length in $O(\log (m \log \sigma))$. As we have $d \in O\left(\log ^{2} n\right)$ and are only storing BWTs of length $m \leq n$ we are storing at most $O\left(\frac{m}{\log ^{2} n}\right)$ pointers. In total these require space $O\left(\frac{m}{\log n}\right)$ bits. When the BWT is coded in this way we can start decoding it at any index which is a multiple of the block size $d \in O\left(\log ^{2} n\right)$. The setup procedure takes $O(1)$ time and we can decode each following symbol in time $O(1)$ when performing a sequential scan (see [15,20] for constant time encoding and decoding of Huffman/minimum redundancy code. The code lengths of the $\gamma$ code can be decoded using a lookup table taking $o(n)$ bits).

Dense $\gamma$ coded gap arrays can be stored very similar to our BWT storage scheme for allowing the start of a decoding run from any position in the array with very little overhead. As shown above an array $G$ of length $l$ such that $\sum_{i=0}^{l-1} G[i]=s$ can be stored using $O(s+l)$ bits, which means the start index for the code of any position can be stored in $O(\log (s+l))$ bits. We decompose the array into non-overlapping blocks of length $e \in O\left(\log ^{2} n\right)$ elements and store a pointer to the start of each block. These pointers take space $O\left(\frac{s+l}{\log n}\right)$ bits in total and we can start the decoding from any index on $G$ after a setup time in $O\left(\log ^{2} n\right)$. Subsequently each following element can be sequentially decoded in constant time per element.

For the sparse $\gamma$ coded gap files we have already described the code itself above. Let us assume we are considering a gap array $G$ of length $\ell$ such that the sum over the elements of $G$ is $s$ and $G$ contains $k$ non-zero values. To facilitate 
the start of a decoding run at a given index on $G$ we keep the $\gamma$ code as is, where we assume that the two $\gamma$ coded sequences (distance between non-zero values in the array and non-zero values) are stored interleaved. As the array is sparse we possibly would be adding excessive amounts of space by adding pointers marking the next non-zero value for equidistant indices on $G$. Instead we store information for every $j=\lceil\log n\rceil^{2}$ 'th instance of a non-zero value on $G$ for $i=0,1,\left\lfloor\frac{k}{j}\right\rfloor$. For each such instance of a non-zero value we store the bit position of the code pair in the $\gamma$ code and the absolute index on $G$. The $\gamma$ code itself requires $O\left(k\left(1+\log \frac{s \ell}{k^{2}}\right)\right)$ bits as shown above, so each pointer into the code takes $O(\log (k \log n))$ bits and there are $O\left(\frac{k}{\log ^{2} n}\right)$ such pointers, so the space used for pointers is $O\left(\frac{k \log (k \log n)}{\log ^{2} n}\right)$ bits which is bounded by the space used for the sparse array itself. Storing the selected absolute values of non-zero indices on $G$ takes space $O\left(\frac{k}{\log n}\right)$. Using this index on the external memory representation for $G$ we can start decoding the array from any index on $i$ on $G$ by first employing a binary search on the absolute value of the largest indexed non-zero value element in $G$ with an index not greater than $i$. This element may be $O\left(\log ^{2} n\right)$ non-zero elements away from our desired target which need to be skipped before we reach the relevant portion of $G$. Thus the setup time is $O\left(\log ^{2} n\right)$. After the setup we can decode either the sequence of non-zero values starting from $i$ or the sequence of all values starting from $i$ in constant time for each such value. Decoding non-zero values is required for merging two sparse arrays while decoding all values is required for merging two BWTs.

For parallel encoding of these data structures we note that all of them are easily writable in any given number of parts to a set of files such that a simple combination of these files represents the respective complete structure. Thus encoding the data structures does not need be performed sequentially. If we write data to multiple files, then we need a simple additional layer of indexing meta data designating the length of the corresponding sequence in each file. For $g t$ bit vector, Burrows-Wheeler transform and dense $\gamma$ coded gap files this additional data stores the number of bits, symbols and gap array entries respectively in the form of a prefix sum array. This array allows us to find the correct starting file and offset in this file given the offset in the complete sequence in logarithmic time in the number of files. The number of files for any one such sequence is bounded by the number of processors, so the space for the prefix sum array is not a concern as long as the number of processors $p$ is $O\left(\frac{n}{\log n}\right)$. For sparse $\gamma$ coded gap files the situation is slightly more involved, as we want to be able to start processing from a given index $i$ on the gap file to process the entry at index $i$ and all gap file entries following $i$ or given some $j$ skip $j$ non-zero values from the front of the file and process all following nonzero elements. For this purpose we store two index sequences in addition. The first is a prefix sum sequence over the number of gap array elements represented by each file. Here the number stored in a file is obtained as

- the length of the gap array if there is only one file or

- otherwise, if there is more than one file

- the index of the last non-zero gap array value represented in the file plus one for the first file,

- the index of the last non-zero gap array value represented in the current file minus the index of the last non-zero gap array value represented in the previous file if the file is neither the first nor the last and

- the length of the full gap array minus the index of the last non-zero gap array value represented in the penultimate file for the last file.

The second is the prefix sum sequence over the sequence of non-zero values stored in each file. Both additional index sequences store $O(p)$ values. Again the space for these can be neglected when $p \in O\left(\frac{n}{\log n}\right)$. Searching the file level indexes for the correct file and in file offset for a given global offset in each case takes time $O(\log p)$ and is thus not critical in comparison with the in file access times for BWT and gap files. We will thus without loss of generality below discuss a situation were the respective streams are assumed to be given as a single file instead of a set of files for the sake of simplicity of exposition. 


\subsection{Parallel Construction of Rank Indexes}

There are several data structures which allow constant time rank queries on sequences over a finite alphabet. In this paper we will consider the wavelet tree (see [10]). A prefix free code $g$ for an alphabet $\Gamma$ is a function $g: \Gamma \mapsto\{0,1\}^{*}$ such that for all pairs of symbols $(a, b) \in \Gamma^{2}$ such that $a \neq b$ the code $g(a)$ is not a prefix of $g(b)$. In the following we in addition assume that each prefix free code considered is such that each binary string $c$ either has a prefix which is a code of some symbol or $c$ is a prefix of at least one code produced by the code. This condition implies that there are no unused binary code words. In consequence the longest code assigned to any symbol is bounded by the size of the input alphabet. For any prefix free code $g: \Gamma \mapsto\{0,1\}^{*}$ where $|\Gamma|>1$ let $g_{i}: \Gamma_{i} \mapsto\{0,1\}^{*}$ denote the sub code obtained from $g$ by choosing $\Gamma_{i}=\{a \in \Gamma \mid g(a)[0]=i\}$ and $g_{i}(a)=g(a)[1,|g(a)-1|]$. Informally $g_{i}$ is defined for all symbols in $\Gamma$ for which the first bit of the code assigned by $g$ is $i$ and this first bit is stripped off when transforming $g$ to $g_{i}$. Let $f_{i}: \Gamma^{*} \mapsto \Gamma_{i}^{*}$ be the function given by the homomorphism defined by mapping each symbol in $\Gamma_{i}$ to itself and all other symbols to the empty word, i.e. the function removing all symbols which are not in $\Gamma_{i}$. Let $h: \Gamma \mapsto\{0,1\}^{*}$ denote a prefix free code. Then the wavelet tree concerning $h$ for a text $s \in \Gamma^{*}$ is obtained in the following recursive way.

- If $|\Gamma|=1$ then produce a leaf.

- Otherwise store the bit sequence $h\left(s_{0}\right)[0] h\left(s_{1}\right)[0] \ldots h\left(s_{|s|-1}\right)[0]$ in an inner node. Attach a left and right child to this inner node where the left child is the wavelet tree for $f_{0}(s)$ concerning the code $h_{0}$ and the right child is the wavelet tree for $f_{1}(s)$ concerning the code $h_{1}$.

When this procedure is complete, then we assign a binary code to each inner node, where we assign $\epsilon$ to the root, $c 0$ to each inner node which is a left child of a parent with code $c$ and $c 1$ to each inner node which is a right child of a parent with code $c$. For the parallel construction of a wavelet tree based on a prefix free code $h$ from an input string observe that each interval of indices on the input string corresponds to a unique interval of indices in the bit vector stored in any inner node of the wavelet tree. For constructing a wavelet tree in parallel from an input string $s$ of length $m$ over $\Sigma$ using $p$ processors we use the following steps.

1. Choose a block size $d=\left\lfloor\frac{m+p-1}{p}\right\rfloor$.

2. Assign index block $I_{i}=[d i, \min (d i+d, m))$ to processor $i$.

3. Each processor computes a symbol histogram $H_{i}: \Sigma \mapsto \mathbb{N}$ for its interval such that $H_{i}[a]=\mid\{j \mid j \in$ $I_{i}$ and $\left.s[j]=a\right\} \mid$.

4. Compute the prefix sum arrays $\hat{H}_{i}$ defined by $\hat{H}_{i}[a]=\sum_{j=0}^{i-1} H_{i}[a]$ for all $a \in \Sigma$. This can be parallelised along the alphabet $\Sigma$.

5. Let $C(h)=\{h(a) \mid a \in \Sigma\}$ and let $\hat{C}(h)=\left\{c^{\prime} \mid c^{\prime}=c[0, i]\right.$ for some $c \in C(h)$ and $\left.-1 \leq i<|c|-1\right\}$ (the set of all proper prefixes of codes produced by $h)$. Let $P_{i}: \hat{C} \mapsto \mathbb{N}$ be defined by $P_{i}(j)=\sum_{k \mid j \text { prefix of h(k) }} \hat{H}(k)$. Processor $i$ computes table $P_{i}$. Note that $P$ may be understood as a function from integers to integers instead of binary strings to integers, as the elements of $\hat{C}$ may be interpreted as the numerical value represented by the respective binary strings. $P_{i}$ denotes the bit offset of the input interval for input block $i$ for each inner node of the constructed wavelet tree.

6. Processor $i$ produces the wavelet tree for interval $I_{i}$ while instead of starting at the beginning of the bit vector of a node starting at the bit position designated by the array $P_{i}$.

7. For each node of the wavelet tree the $p$ processors compute the necessary prefix sums for the binary rank dictionaries of each node in parallel.

This approach can be used to obtain a rank dictionary for the input string $s$ of length $m$ over $\Sigma$ in time $O\left(\frac{m}{p}\right)$ using $p$ processors. This applies for block type as well as Huffman code. 


\subsection{Parallel Computation of Gap Arrays}

When we compute a gap array we are generally merging two adjacent text blocks. Let the left and right block sizes be $b_{l}$ and $b_{r}$ respectively and let the indices of the left block on $\tilde{t}$ start at index $i$. In the serial version we start with the suffix at index $i+b_{l}+b_{r}-1$ and perform $b_{r}-1$ steps of backward search using the index for the left block. This can be parallelised by performing several backward searches in parallel starting from several indices within the right block. If $p \leq b_{r}$ processors are available, then we can choose the starting positions $x_{j}=i+b_{l}+b_{r}-1-j\left\lceil\frac{b_{r}}{p}\right\rceil$ for $j=0,1, \ldots, p-1$ and perform $\ell_{j}=x_{j+1}-x_{j}$ steps for $j=0,1, p-2$ and $\ell_{j}=b_{r}-x_{j}$ steps for $j=p-1$. As the backward search is based an suffix ranks in the left block we need to find the ranks of these $p$ suffixes in the left block using forward search on one or more suffix arrays just as we did above for the single suffix at the end of the right block in serial mode. As we have $p$ processors and need to find $p$ ranks, the run-time stays the same as in the serial version for both average and worst case. In the average case the forward searches are not a time critical factor as long as $p$ is $O\left(\frac{n}{\log ^{2} n \log \log n}\right)$, so in practice we can expect the forward searches to be of no concern for the execution time. When we perform $p$ backward searches in parallel, then we also need to be prepared to perform the corresponding increment operations on the gap array in parallel. This is no problem if the gap array can be kept in internal memory as modern processors have specialised operations for atomic, lock free modifications of single memory cells. If we use sparse gap arrays in external memory, then the situation is somewhat more complicated. In the serial version we first accumulate a number of indices in the gap array whose value is to be incremented in a list of length $k \in O\left(\frac{b_{r}}{\log ^{2} b_{r}}\right)$ in internal memory. If this list runs full, then it needs to be sorted and written to external memory as a sparse $\gamma$ coded gap array. Whenever there are two sparse gap arrays of the same sum, then the two need to be merged to obtain a single sparse array of twice that sum (as the number of non-zero elements in the files approaches the size of the left block we switch to dense arrays as described above. Generating and merging dense $\gamma$ coded gap arrays are however simplified versions of the equivalent problems in sparse arrays, so we will not explicitly discuss this below as the algorithms are easily deduced from the sparse case). In the parallel case we keep the concept of a single array accumulating indices in internal memory. All $p$ processors are appending values to this array until it runs full. This can be implemented by using a fill pointer which is modified by atomic increments while elements are inserted at the array index designated by the fill pointer before the increment. A process stalls backward search when the pointer exceeds a given threshold signalling that the array is full. When all processes have detected that the array is full, then we can perform a parallel radix sort on the array. As in the serial case this is performed in two stages, i.e. using $e \in O(\sqrt{n})$ buckets. Like for histogram computation in the parallel wavelet tree construction we partition the array into $p$ non overlapping intervals and compute a separate histogram for each interval. This takes space $O(p \sqrt{n} \log n)$ bits which is negligible as long as $p \in o\left(\frac{\sqrt{n}}{\log n}\right)$ which is not an issue in practice. Prefix sums are computed based on these bucket histograms to obtain the starting index for the elements for each bucket originating from each processor input interval. The elements for bucket $i$ appear ahead of those for buckets with index larger than $i$ and within each bucket the elements originating from processor interval $j$ appear before those for intervals with higher indices. These prefix sums can be computed in parallel by first accumulating all element counts over $\left\lceil\frac{e}{p}\right\rceil$ buckets and then based on these offsets filling in the final prefix sums in parallel. Using the computed prefix sums the numbers can be sorted into their buckets in parallel while keeping the stable order necessary for a correct radix sort. Using this radix sort method we can sort a set of the $k$ numbers from $\left[0, b_{l}\right]$ in time $O\left(\frac{\max \left\{\sqrt{b_{l}}, k\right\}}{p}\right)$ using $p$ processors. After the array is sorted we again partition it into $p$ index intervals. In each interval in parallel we transform the now sorted sequence into run length encoding, i.e. pairs of numbers and the number of occurrences of the number in the interval. This representation does not asymptotically need more space than the sorted number sequence. The computation of the run length encoding in intervals produces a correct run length encoding of the complete sequence except for the borders of the intervals. Here the last run length in one interval may be for the same number as the first run length in the next interval. This however can be corrected in time $O(\log p)$ using $p$ processors, where we assign the complete run-length to the leftmost occurrence of the number and mark the other occurrences as invalid. After this we can produce a compact version without space between the intervals of the run length encoded array in internal memory in time $O\left(\frac{k}{p}\right)$ by counting the number of 
runs per interval, computing the prefix sums over this number sequence and copying the sequence to another array in compact form. Finally we decompose this compact run length array into at most $p$ intervals of length $\left\lceil\frac{\left\lceil\frac{k}{p}\right\rceil}{e}\right\rceil e$ where $e \in O\left(\log ^{2} n\right)$ is a positive integer and write the resulting decomposition to $p$ sparse $\gamma$ coded gap arrays such that the concatenation of the files represents the complete sparse gap array. As each interval size but the last is a multiple of $e$ each file starts on an index of the sequence of run length pairs which is also a multiple of $e$. This file production also takes time $O\left(\frac{k}{p}\right)$. Finally we can also produce the block pointers required for accessing the file without performing a linear scan in the same time complexity. Summarising we can transform the array of length $k$ accumulating indices for incrementing in the gap array to a sparse $\gamma$ coded representation in external memory in time $O\left(\frac{k+\sqrt{b_{r}}}{p}\right)$ using $p$ processors. Now assume we have two sparse $\gamma$ coded gap arrays with $k_{a}$ and $k_{b}$ non-zero values respectively which are to be merged. We want to perform this merging using $p$ processors, where in the optimal case each processor is assigned the same amount of work. Both files store $e \in O\left(\log ^{2} n\right)$ non-zero values per block. Using a binary search on these values we can determine the smallest $i$ such that at least $\left\lceil\frac{k_{a}+k_{b}}{p}\right\rceil$ blocks are stored in the two files for values smaller than $i$. The first processor merges the values of the two input sequences from the beginning up to value $i$. The same scheme is used to split the remaining data into a work package for the second processor and the rest for the remaining processors etc. The splitting operations take time $O\left(p \log ^{3} n\right)$. We are computing $p$ splitting points, each such splitting point is computed using a binary search on $O(n)$ sorted values and accessing each such value takes time $O\left(\log ^{2} n\right)$. After the splitting the merging is easily done in parallel in time $O\left(\frac{k_{a}+k_{b}}{p}\right)$ so the run time of the method is $O\left(p \log ^{3} n+\frac{k_{a}+k_{b}}{p}\right)$. This is a convenient procedure when the number of processors is low compared to the size of the input. If the number of processors should not be neglectable in comparison to the input size, i.e. if $O\left(p \log ^{3} n\right)$ would be large, then we could employ a different splitting method where we would decompose the merging work along an optimally balanced binary tree and thus reduce the work for finding the splitting points to $O\left(\log p \log ^{3} n\right)$. We would still be computing $O(p)$ splitting points, but the splitting point search within each level of the tree of depth $O(\log p)$ could be performed in parallel in time $O\left(\log ^{3} n\right)$.

\subsection{Merging Burrows-Wheeler Transforms}

For merging two BWT sequences of length $b_{l}$ and $b_{r}$ respectively let us assume we have the two sequences in external memory and the gap array in internal or external memory. For parallel merging of the two sequences we first compute the sum over the gap array for each interval of integer length $e \in O\left(\log ^{2} n\right)$ starting at index $i e$ on the gap array $G$ for $i=0,1, \ldots, \frac{b_{l}}{e}$ using $p$ processors in parallel in time $O\left(\frac{b_{l}}{p}\right)$. These can be produced sequentially and in external memory and take space $o\left(b_{l}\right)$ bits. Then we compute the prefix sums over this sequence in parallel in time $O\left(\frac{b_{l}}{p}\right)$ and also store it in external memory. Using these prefix sums we can assign approximately equal work loads to each processor. Using a binary search on the prefix sums we can search for the smallest $j<b_{l}+1$ such that $s=\sum_{i=0}^{j}(G[i]+1) \geq\left\lceil\frac{b_{l}+b_{r}}{p}\right\rceil$ and then increase $j$ and update the sum $s$ accordingly until $\left\lceil\frac{s}{e}\right\rceil e-s \leq G[j+1]$ or $j=b_{l}+1$ if no such $j$ exists (remember that $G$ has length $b_{l}+1$ ). The binary search gives us a work package which is approximately $\frac{1}{p}$ of the total length. The subsequent increasing of $j$ is for allowing us to produce a file with a length which is a multiple of the block size by just considering the next gap array value $G[j+1]$. The case where there is no such $j$ can only occur for very sparse arrays with high run length values. The first processor merges the front parts of the two BWTs by in turn taking $G[i]$ symbols from the second BWT stream and one symbol from the first one for $i=0,1, \ldots, j$ and finally $\left\lceil\frac{s}{e}\right\rceil e-s$ from the second BWT stream to obtain a multiple of the block size $e$. The rest of the two BWT streams is merged by the other processors. The further splitting of the remaining data into blocks is performed just like the splitting of the data into the part for the first processor and the rest. The only difference consists in the fact that a fraction of $G[j+1]$ is already handled by the first processor and thus needs to be excluded from the remaining process. As for merging sparse $\gamma$ coded gap arrays the finding of the splitting points in this manner takes time $O\left(p \log ^{3} n\right)$ following a similar argumentation. The actual merging is then performed in time $O\left(\frac{b_{l}+b_{r}}{p}\right)$ using $p$ processors. In practice it may be desirable to perform the merging directly using the run length encoding instead of symbol per symbol. In the asymptotical worst case there is however no difference in the 
run time. Like in the case of merging sparse $\gamma$ coded gap files we can speed up the computation of the splitting points to $O\left(\log p \log ^{3} n\right)$ if necessary. The approach of parallelisation of BWT merging can be extended to multi way instead of two way merging as described in [12]. The concepts remain the same, but the searching of split points is over all of the involved prefix sum arrays of gap files instead of just one. The merging within the single merge packets remains the same as in the serial version.

\subsection{Parallel Sorting of Single Blocks}

We need to suffix sort single blocks and produce the respective block BWTs before we can merge these structures to obtain the final BWT of the complete text. To our best knowledge there is currently no parallel in place suffix sorting algorithm. The external memory algorithmic variants described in [14] either distribute a total work of $\Omega(n \log n)$ over $p$ processors or work in some randomised model of computation. As the problem of parallel suffix sorting in place is still open, we resort to using our parallel block merging scheme to achieve some parallelism for the average case. In the highly unlikely worst case this will again not bring any improvement over the serial version. The block size $b$ in the serial version is chosen to allow the suffix sorting of a single block in internal memory. For the parallel case we deduce a reduced block size $b_{p}=\left\lceil\frac{b}{p}\right\rceil$ which allows us to perform the suffix sorting of $p$ such blocks at the same time in internal memory. After these blocks have been handled we merge the resulting BWTs until we obtain the BWTs for block size $b$ using our parallel merging approach described above. Note that this does not require us to store gap arrays in external memory, as we have sufficient space in internal memory to perform the merging. In the unlikely worst case we will spend time $\Omega(n \log n \log p)$ for finding the start ranks of our backward searches during gap array construction. On average the run time is $O\left(\frac{b \log p}{p}\right)$ in all practically relevant cases (i.e. when $\left.p \log p \in O\left(\frac{n}{\log ^{4} n}\right)\right)$.

\subsection{Parallelisation of the Complete Algorithm}

Using the building blocks described above we can now present the parallel version of our algorithm for $p$ processors. The steps are:

1. Choose a target block size $b^{\prime \prime} \in O\left(\frac{n}{\log n}\right)$ as in the serial version. Transform this block size $b^{\prime \prime}$ to $b^{\prime}=\left\lceil\frac{b^{\prime \prime}}{p}\right\rceil$, a target block size for parallel computing and finally deduce a block size $b=\left\lceil\frac{n}{\left\lceil\frac{n}{b^{\prime}}\right\rceil}\right\rceil$. Split the text $t$ into blocks of size $b$ and $b-1$ only such that all the blocks of length $b$ appear before the ones for $b-1$. This step takes constant time and space.

2. Compute which blocks in $\tilde{t}$ propagate repetitions of a period at most $b$ and for each block which is followed by a block propagating a repetition whether it is generating this repetition. We can handle $p$ blocks in parallel during the propagation as well as the generation checking phase as each block can be handled independently and we have sufficient space in internal memory due to the reduced block size compared to the serial version. This step consequently takes time $O\left(\frac{n}{p}\right)$ in total and space $O\left(p \frac{n \log \sigma}{\log n p}\right)=O\left(\frac{n \log \sigma}{\log n}\right) \subseteq O(n)$ bits. The result can be stored in external memory.

3. Compute a balanced merge tree for the blocks in serial like for the serial cases. This takes time $O(\log n)$ and space $o(n)$ bits.

4. Sort the blocks and store the resulting BWT, $g t$ and sampled inverse suffix arrays in external memory while processing $p$ blocks at a time. Using the suffix and LCP arrays of the basic blocks also compute the start ranks necessary for the backward searches when merging blocks together. This step takes time $O(n \log n(\log \log n+$ $\log p))$ in the worst case and $O\left(\frac{n}{p}\right)$ on average and space $O\left(b^{\prime} \log b^{\prime}\right)=O(n)$ bits of internal memory.

5. Process the merge tree. Mark all leafs as finished and all inner nodes as unfinished. While there are unfinished nodes choose any unfinished node with only finished children, merge the respective blocks and mark the node as finished. When merging blocks use an internal memory gap array if the node has a distance of at $\operatorname{most}\lceil\log p\rceil$ 
from any leaf in the underlying sub tree and sparse $\gamma$ coded gap arrays in external memory otherwise. Use the parallel versions of gap array computation, merging of $\gamma$ coded gap array files, merging of BWT streams and merging sampled inverse suffix array files. The $\log \log n$ levels closest to the root can be processed in total time $O\left(\frac{n \log \log n}{p}\right)$ per level or $O\left(\frac{n \log ^{2} \log n}{p}\right)$ in total. The $\log p$ other (lowest) levels of the tree can be processed in time $O\left(\frac{n \log p}{p}\right)$. The total run-time for the merging stage is thus $O\left(\frac{n}{p} \max \left\{\log ^{2} \log n, \log p\right\}\right)$. The space requirement in internal memory remains asymptotically the same as in the serial version as long as $p \in o\left(\frac{\sqrt{n}}{\log n}\right)$ as described above.

The total run-time of the parallel algorithm using $p$ processors is thus $O\left(\frac{n}{p} \max \left\{\log ^{2} \log n, \log p\right\}\right)$ on average and with high probability and $O(n \log n(\log \log n+\log p))$ in the worst case. As in the serial case we can resort to the original skewed merging approach on top of the balanced merging if there is not sufficient internal memory to hold the index for the left half of the text in memory but sufficient to keep an index for a fixed fraction of the text while keeping the same run-time and space bounds.

\section{Conclusion}

We have presented a new semi external algorithm for computing the Burrows-Wheeler transform designed for the case where we can keep a fixed fraction of the input text in internal memory. On average our new algorithm runs in time $O\left(n \log ^{2} \log n\right)$ and is faster then the algorithm of Ferragina et al. [8] while in the worst case it is only slower by a factor of $O(\log \log n)$. In comparison with the algorithm by Beller et al. [1] our algorithm can be applied for the case when less than 8 bits per symbol of internal memory are available. We have also presented a parallel version of our algorithm which on average and with high probability runs in time $O\left(\frac{n}{p}\left(\log ^{2} \log n+\log p\right)\right)$ on $p$ processors and should achieve this run time in all practically relevant applications. In the worst case the run time of the parallel algorithm is $O(n \log n(\log \log n+\log p))$ and thus, depending on the number of processors $p$, possibly even slower than the serial version. However this worst case is highly unlikely in practice.

The practically interested reader can find a partial implementation of the algorithms presented in this paper at https://github.com/gt1/bwtb3m. The current version of the code does not implement the repetition reduction as described in Sect. 5 but uses a simplified approach computing the length of the longest common prefix of all suffixes in a block with the first suffix of the following block for determining how far a block needs to be extended to obtain a correct sorting order. On average this extension is by $O(\log n)$ characters. For texts featuring long repetitions of short periods (shorter than the block size) however the method would be problematic.

One major open problem in our approach is the worst case run time. While it is not as extreme as the worst case of some other algorithms for suffix sorting or BWT construction showing good average case behaviour (cf. [24]), it still leaves room for improvement. This is particularly true for the case of the parallel variant of the algorithm. The increased worst case run-time over the average case stems solely from the forward searches on block suffix arrays to obtain the starting points for the backwards searches during gap array construction. In the serial version a string needs to feature a very large LCP value in $\Omega\left(\frac{n \log \log n}{\log n}\right)$ between two of its suffixes to trigger the worst case behaviour, in the parallel case using $p$ processors a LCP value in $\Omega\left(\frac{n \log \log n}{p \log n}\right)$ is sufficient. So an efficient method for detecting such high LCP values using small space would be necessary to alleviate the effects of the problem. Note that these high LCP values are not necessarily caused by runs (cf. [18]) as the positions of the suffixes in the string sharing a long common prefix may be too far apart to allow the common strings to connect, i.e. build a square.

Two other open problems are the actual practical space usage of our algorithm in external memory and its $\mathrm{I} / \mathrm{O}$ complexity. While the space usage in external memory is asymptotically clear with $O(n \log \sigma)$ bits the involved constants are important in practice. Punctual observations in our implementation studies suggest that the involved constants are low, but a systematic analysis is desirable. A very simple analysis in analogy to the space usage shows that the algorithm uses $O\left(n \log ^{2} \log n\right)$ bits of I/O for merging $\gamma$ coded gap arrays, $O(n \log \sigma \log \log n)$ bits of I/O for merging BWTs and $O(n \log \log n)$ bits of I/O for writing and reading $g t$ arrays, where I/O is in all 
cases streaming. As for the space usage in external memory, it would also be interesting to determine the involved constants in future work.

Acknowledgements Open access funding provided by Max Planck Society.

Open Access This article is distributed under the terms of the Creative Commons Attribution 4.0 International License (http:// creativecommons.org/licenses/by/4.0/), which permits unrestricted use, distribution, and reproduction in any medium, provided you give appropriate credit to the original author(s) and the source, provide a link to the Creative Commons license, and indicate if changes were made.

\section{References}

1. Beller, T., Zwerger, M., Gog, S., Ohlebusch, E.: Space-efficient construction of the Burrows-Wheeler transform. In: Kurland, O., Lewenstein, M., Porat, E. (eds.) SPIRE. Lecture Notes in Computer Science, vol. 8214, pp. 5-16. Springer, Berlin (2013)

2. Bingmann, T., Fischer, J., Osipov, V.: Inducing suffix and LCP arrays in external memory. In: Sanders, P., Zeh, N. (eds.) ALENEX, pp. 88-102. SIAM, Philadelphia (2013)

3. Burrows, M., Wheeler, D.: A block-sorting lossless data compression algorithm. Digital Systems Research Center, RR-124 (1994)

4. Crochemore, M., Grossi, R., Kärkkäinen, J., Landau, G.M.: A constant-space comparison-based algorithm for computing the Burrows-Wheeler transform. In: Fischer, J., Sanders, P. (eds.) CPM. Lecture Notes in Computer Science, vol. 7922, pp. 74-82. Springer, Berlin (2013)

5. Crochemore, M., Hancart, C., Lecroq, T.: Algorithms on Strings. Cambridge University Press, Cambridge (2007)

6. Dementiev, R., Kärkkäinen, J., Mehnert, J., Sanders, P.: Better external memory suffix array construction. ACM J. Exp. Algorithmics 12, 1-24 (2008)

7. Elias, P.: Universal codeword sets and representations of the integers. IEEE Trans. Inf. Theory 21(2), 194-203 (1975)

8. Ferragina, P., Gagie, T., Manzini, G.: Lightweight data indexing and compression in external memory. Algorithmica 63(3), 707-730 (2012)

9. Ferragina, P., Manzini, G.: Opportunistic data structures with applications. In: 41st Annual Symposium on Foundations of Computer Science, 2000. Proceedings, pp. 390-398. IEEE, New York (2000)

10. Grossi, R., Gupta, A., Vitter, J.S.: High-order entropy-compressed text indexes. In: SODA, pp. 841-850 (2003)

11. Hon, W.-K., Sadakane, K., Sung, W.-K.: Breaking a time-and-space barrier in constructing full-text indices. In: FOCS, pp. 251-260. IEEE Computer Society, New York (2003)

12. Kärkkäinen, J., Kempa, D.: Engineering a lightweight external memory suffix array construction algorithm. In: Iliopoulos, C.S., Langiu, A. (eds.) 2nd International Conference on Algorithms for Big Data (ICABD2014), number 1146 in CEUR-WS Proceedings, pp. 53-60, Aachen (2014)

13. Kärkkäinen, J., Sanders, P.: Simple linear work suffix array construction. In: Automata, Languages and Programming, pp. $943-955$. Springer, Berlin (2003)

14. Kärkkäinen, J., Sanders, P., Burkhardt, S.: Linear work suffix array construction. J. ACM 53(6), 918-936 (2006)

15. Kärkkäinen, J., Tischler, G.: Near in place linear time minimum redundancy coding. In: Bilgin, A., Marcellin, M.W., Serra-Sagristà, J., Storer, J.A. (eds.) DCC, pp. 411-420. IEEE, New York (2013)

16. Kasai, T., Lee, G., Arimura, H., Arikawa, S., Park, K.: Linear-time longest-common-prefix computation in suffix arrays and its applications. In: Amir, A., Landau, G.M. (eds.) CPM. Lecture Notes in Computer Science, vol. 2089, pp. 181-192. Springer, Berlin (2001)

17. Knuth, D.E., Morris Jr., J.H., Pratt, V.R.: Fast pattern matching in strings. SIAM J. Comput. 6(2), 323-350 (1977)

18. Kolpakov, R., Kucherov, G.: Finding maximal repetitions in a word in linear time. In: Proceedings of the 1999 Symposium on Foundations of Computer Science (FOCS'99), New York, USA, pp. 596-604, 17-19 October 1999. IEEE Computer Society, New York (1999)

19. Manber, U., Myers, G.: Suffix arrays: a new method for on-line string searches. SIAM J. Comput. 22(5), $935-948$ (1993)

20. Moffat, A., Turpin, A.: On the implementation of minimum redundancy prefix codes. IEEE Trans. Commun. 45(10), 1200-1207 (1997)

21. Navarro, G., Mäkinen, V.: Compressed full-text indexes. ACM Comput. Surv. (CSUR) 39(1), 2 (2007)

22. Nong, G., Zhang, S., Chan, W.H.: Two efficient algorithms for linear time suffix array construction. IEEE Trans. Comput. 60(10), 1471-1484 (2011)

23. Okanohara, D., Sadakane, K.: A linear-time Burrows-Wheeler transform using induced sorting. In: Karlgren, J., Tarhio, J., Hyyrö, H. (eds.) SPIRE. Lecture Notes in Computer Science, vol. 5721, pp. 90-101. Springer, Berlin (2009)

24. Puglisi, S.J., Smyth, W.F., Turpin, A.H.: A taxonomy of suffix array construction algorithms. ACM Comput. Surv. 39(2), 1-31 (2007)

25. Szpankowski, W.: On the height of digital trees and related problems. Algorithmica 6(1-6), 256-277 (1991) 